\title{
Reoccurrence of Levofloxacin-Induced Tendinitis by Phenoxymethylpenicillin Therapy after 6 Months: A Rare Complication of Fluoroquinolone Therapy?
}

\author{
Christoph Schindler David Pittrow Wilhelm Kirch \\ Institute of Clinical Pharmacology, Medical Faculty of the Dresden University of Technology, Dresden, Germany
}

Levofloxacin has been characterized as safe and unlikely to cause severe adverse events. Ball et al. [1] reported that adverse events associated with fluoroquinolones range from $0.5 \%$ to almost $7 \%$. New second-generation fluoroquinolones such as levofloxacin have an extended spectrum of antimicrobial activity compared with older substances but a similar safety profile.

The potential of fluoroquinolones to induce tendinitis and even tendon rupture, usually of the Achilles tendon, is low but well documented [2-7]. Although different pathophysiological hypotheses are discussed, the mechanisms of this effect are poorly understood. Studies performed on rats suggest a toxic effect of fluoroquinolones on cellular components of connective tissue structures [2]. Lesions experimentally induced in rats by fluoroquinolone treatment were characterized by edema and mononuclear cell infiltration in the inner sheath of the Achilles tendon, with infiltration into the adjacent synovial membrane and joint space. Cellular pathological effects observed in the cartilage and tendons show many identical features, indicating that quinolone-induced arthropathy and tendopathy are probably different clinical manifestations of the same toxic effect on cellular components of connective tissue structures [2]. However, the impact of additional factors, if any, in determining susceptibility to such adverse effects remains unclear.
Symptoms usually resolve within a few weeks, but in $10 \%$ of patients they may persist for several months. Pharmacovigilance data revealed that tendinitis occurred in 1/500,000 levofloxacin prescriptions, with tendon ruptures only in 1/1,6 million prescriptions [3], but there are probably a considerable number of unidentified cases. Tendinopathy has been most frequently reported with perfloxacin, but also occurs with levofloxacin [4]. We describe for the first time a patient with levofloxacin-associated tendinitis presenting with reoccurring symptoms induced by phenoxymethylpenicillin therapy 6 months after stopping levofloxacin treatment.

A 65-year-old nonfebrile asymptomatic woman was prescribed levofloxacin $500 \mathrm{mg} /$ day for antibiotic treatment of Yersinia-reactive arthritis in August 2001. After day 10 of daily treatment with $500 \mathrm{mg}$ levofloxacin, the patient developed a painful inflammatory tendinopathy of the Achilles tendon of the right leg. Levofloxacin was stopped immediately and the symptoms resolved within 3 weeks after immobilization of the Achilles tendon.

The patient has a history of chronic bronchial asthma and has been on oral low-dose steroids for 24 years, a known risk factor for developing fluoroquinolone-induced tendinitis. In February 2002, phenoxymethylpenicillin 600,000 IE (3 tablets) a day was prescribed after dental surgery. After taking the second dose of penicillin,
Christoph Schindler, MD

Institute of Clinical Pharmacology, Medical Faculty, Dresden University of Technology Fiedlerstrasse 27, D-01307 Dresden (Germany)

Tel. +49 351458 2027, Fax +49 3514584341

E-Mail christoph.schindler@mailbox.tu-dresden.de 
the patient developed again tendinopathy of the Achilles tendon of the right leg with painful induration, very similar to the clinical manifestation induced by levofloxacin. Penicillin was discontinued and therapy was switched to clindamycin which was given for another 4 days. The patient's subjective discomfort improved rapidly within $48 \mathrm{~h}$ after penicillin had been discontinued. After placing the Achilles tendon at rest, the symptoms of tendinitis completely resolved within 3 weeks.

We informed the German Federal Institute for Drugs and Medical Devices and the Drug Commission of the German Medical Profession. According to their database, no other cases of levofloxacin-associated tendinitis reoccurring after phenoxymethylpenicillin-therapy have ever been reported.

Age, renal dysfunction, magnesium deficiency and long-time steroid therapy have been identified as risk factors for quinolone-associated tendinitis [5]. Therefore, some authors even recommend avoiding fluoroquinolones in elderly patients, especially those receiving higher doses of corticosteroid therapy [6]. In very rare instances, and for unknown reasons, patients who already experi- enced a tendinopathy after levofloxacin may be at risk of suffering a recurrence of their tendinitis following penicillin, especially if they exhibit additional risk factors. Nevertheless, the mechanisms of tendinitis as a class-related side effect of fluoroquinolones remain unclear.

This case report gives rise to the suspicion that a positive history of levofloxacin-associated tendinitis could probably complicate any further antibiotic therapy in predisposed patients. Phenoxymethylpenicillin could act as a trigger for the reoccurrence of tendinitis. As a potential differential diagnosis for our patient's symptoms after phenoxymethylpenicillin therapy we cannot completely exclude the development of enthesitis of the right heel as a reaction to Yersinia infection. However, this would not explain the rapid improvement of symptoms after stopping penicillin therapy and therefore seems unlikely. During therapy with clindamycin, no symptoms of tendinitis reoccurred. This very special case suggests a penicillininduced side effect. Further research is necessary to clarify the pathophysiologic mechanisms of phenoxymethylpenicillin.

\section{References}

1 Ball P, Mandell L, Niki Y, Tillotson G: Comparative tolerability of the newer fluoroquinolone antibacterials. Drug Saf 1999;21:407421.

2 Shakibaei M, Pfister K, Schwabe R, Vormann J, Stahlmann R: Ultrastructure of Achilles tendons of rats treated with ofloxacin and fed a normal or magnesium-deficient diet. Antimicrob Agents Chemother 2000;44:261-266.
3 Carbon C: Comparison of side effects of levofloxacin versus other fluoroquinolones. Chemotherapy 2001;47(suppl 3):9-14.

4 Fleisch F, Hartmann K, Kuhn M: Fluoroquinolone-induced tendinopathy: Also occurring with levofloxacin. Infection 2000;28:256-257.

5 De Sarro A, De Sarro G: Adverse reactions to fluoroquinolones. An overview on mechanistic aspects. Curr Med Chem 2001;8:371-384.
6 Pierfitte C, Royer RJ: Tendon disorders with fluoroquinolones. Therapie 1996;51:419-420.

7 Ribard P, Audisio F, Kahn MF, De Bandt M, Jorgensen C, Hayem G, Meyer O, Palazzo E: Seven Achilles tendinitis including 3 complicated by rupture during fluoroquinolone therapy. J Rheumatol 1992;19:1479-1481. 